\title{
Simulation of fully coupled finite element analysis of nonlinear hydraulic properties in land subsidence due to groundwater pumping
}

\author{
Yong Yang $\cdot$ Xian Fang Song $\cdot$ Fan Dong Zheng • \\ Li Cai Liu · Xiao Juan Qiao
}

Received: 17 January 2014/ Accepted: 13 September 2014/Published online: 23 January 2015

(C) Springer-Verlag Berlin Heidelberg 2015

\begin{abstract}
A fully coupled mathematical model of land subsidence caused by groundwater pumping was established based on the mechanics of porous seepage and the theory of fluid-solid interaction. The mathematical model employing the Galerkin finite element method was proposed to simulate the deformation dependencies of hydraulic properties due to the water pressure decrease in aquifers. This model has been verified by comparing with the known analytical solutions in the confined aquifer. Results show that the simulated drawdown and displacements match well with those of analytical solutions. To evaluate the nonlinear effects of hydraulic properties in the seepage and consolidation coupling phenomena, the numerical model is applied to an ideal three layers numerical experiment. The results show that the subsidence rate is faster than conventional groundwater theory when the nonlinear hydraulic properties (NHP) are considered in the coupled model. The reason is that the water level decline due to groundwater withdrawal induces the soil
\end{abstract}

Y. Yang $(\bowtie) \cdot$ X. F. Song

Key Laboratory of Water Cycle and Related Land Surface

Processes, Institute of Geographic Sciences and Natural

Resources Research, Chinese Academy of Sciences,

Beijing 100101, China

e-mail: steven_yy@163.com

Y. Yang · F. D. Zheng · L. C. Liu

Beijing Water Science and Technology Institute,

Beijing 100048, China

X. J. Qiao

Key Laboratory of Computational Geodynamics, Chinese Academy of Sciences, Beijing 100049, China

X. J. Qiao

College of Earth Sciences, University of Chinese Academy of Sciences, Beijing 100049, China compression and the porosity and permeability decrease. Decrease of permeability in regions adjacent to the pumping well produces hydraulic gradient and seepage forces that may result in accelerated subsidence. Therefore, the effect of the NHP is an interaction process of pore water pressure and soil consolidation deformation and should not be ignored. Further studies of various hydrogeology problems are recommended to consider the heterogeneity concerning different stratigraphic condition in the field.

Keywords Fluid-solid interaction - Land subsidence . Groundwater pumping $\cdot$ Nonlinear $\cdot$ Numerical model

\section{Introduction}

Land subsidence due to groundwater pumping has induced various problems including unequal settlement, wall cracking, pipeline damage and ground fissures in many cities around the world (Khan et al. 2013; Abidin et al. 2013). Groundwater pumping induces the fully coupled hydraulic-mechanical interaction between water flow and solid matrix in nature in many cases. As groundwater pumping take place, pressure decline in aquifers is accompanied by a change in effective stress in the solid matrix, and then compaction of the solid skeleton of the aquifer ensues. But on the other hand, when porous media deformation occurs, its physical parameters, such as porosity, permeability, will also be changed. Therefore, it is necessary to consider the coupling of water flow fluctuations and the deformation characteristics of fluid field media. This fully coupled phenomenon can be better explained through the Biot's consolidation theory which provides a more rigorous and realistic 
mathematical method to analyze the fully coupled groundwater flow and solid matrix deformation. Biot's (1941) consolidation theory has been widely applied in hydrogeology, geomechanics, engineering and many other fields. On the basis of Biot's work, a number of scholars use analytical solutions and numerical solutions to evaluate the fully coupled water flow and solid matrix deformation due to groundwater pumping. Bear and Corapcioglu (1981) proposed an analytical solution for regional land subsidence due to groundwater pumping. Safai and Pinder (1979) constructed a Galerkin finite element model to simulate the vertical and horizontal land deformation in a desaturating porous medium. Schrefler and Zhan (1993) developed a fully coupled model to simulate the slow transient phenomena involving flow of water and air in deforming porous media. Bai and Elsworth (1994) used a dual-porosity poroelastic model to represent the coupled flow and deformation behavior in fractured rock specimens. Yeh et al. (1996) developed a general Galerkin finite element model to study the behavior of land displacements due to pressure decline in aquifers. Gatmiri and Delage (1997) developed a formulation of fully coupled thermal-hydraulic-mechanical behavior of saturated porous media. Lewis and Schrefler (1978, 1998) presented a coupled model to simulate the subsidence and the pressure decline in the considered area. Kim and Parizek (1999) and Kim (2000) presented a fully coupled numerical model for groundwater flow and soil deformation due to groundwater pumping. Gambolati et al. (2000) analyzed the comparison of results obtained with the finite element of the coupled and uncoupled models, which indicated that pore pressure was rather insensitive to the coupling model within the pumped formation. Zimmerman et al. (1986) pointed out that the coupling effect was usually lies between 0.1 and 1.0 and concluded that the pore-elastic coupling effect was usually strong and could not be ignored. These numerical analyses assumed linear coupling between water pressure and solid matrix. However, the hydraulic properties of the porous media depended on the deformation, which induced nonlinear coupling. There have been several experimental and mathematical studies on the permeability and pore-volume changes with pore water pressure and soil stress, such as Zimmerman (2000) believed an increase in temperature may lead to a large increase in fluid pressure, which will then give rise to mechanical stresses and strains. Kim and Parizek (2005) used a fully coupled poroelastic numerical model to simulate the hydraulic and mechanical responses when hydraulic pumping and pumping shutoff, the result showed that poroelastic deformation caused by groundwater pumping and pumping shutoff from an aquifer could induce noticeable reverse hydraulic head fluctuations. Ouria et al.
(2009, 2011) developed a numerical model to investigate the effect of the changes of the soil permeability on the transient seepage, the result showed that the effect of the change of the permeability on the outlet flow was different depending on the coupled or uncoupled formulations. Budhu and Adiyaman (2010, 2013) developed a basic mechanics governing the changes in stress states from groundwater pumping and compared the predicted land subsidence with existing models and field data, the result showed that the predicted subsidence using the developed mechanics compared well with the field data. Results of these studies strongly indicated that the nonlinear character of hydraulic properties should be considered. Some scholars used the fully coupled flow in other fields, for instance, oil reservoirs, coal seams, landfill gas transport, etc (Huang et al. 2013; Zhang et al. 2011; Xue et al. 2005). However, the nonlinear of the hydraulic parameters in parameter dynamic change and the different soil properties were merely considered in those studies. The objective of this paper was to develop a mathematical model which considered the nonlinear of the hydraulic parameters including the variable porosity and permeability and to study the behavior of coupling model of water pore pressure and soil stress. A general Galerkin finite element method was used to solve the numerical solution of coupling model. Finally, the mathematical model was applied to $2 \mathrm{D}$ numerical model experiment with different soil properties to show the effects of nonlinear character of hydraulic properties on consolidation processes.

\section{Governing equations}

A general mathematical model describing the transient flow equation and the equilibrium equation can be derived based on the following assumptions: (1) The medium is an isotropic pore-elastic continuum; (2) fluid flow follows the Darcy's law; (3) the deformation of solid matrix abides by Terzaghi's (1943) law of effective stress

$\sigma_{i j}=\sigma_{i j}^{\prime}+p \delta_{i j}$

where $\sigma_{i j}$ is the tensor of total stress, $\sigma_{i j}^{\prime}$ is the tensor of effective stress, and $p$ is pore pressure, and $\delta_{i j}$ is Kronecker sign.

Equilibrium equation of stress (neglecting the body forces)

$\sigma_{i j, j}=0$.

Geometrical equation

$\varepsilon_{i j}=\frac{1}{2}\left(u_{i, j}+u_{j, i}\right)$.

Constitutive relation of strain and stress 
$\sigma_{i j}^{\prime}=2 G \varepsilon_{i j}+\lambda \varepsilon_{v} \delta_{i j}$

where $G=E / 2(1+v)$ is the shear modulus of the porous medium, $E$ is Yang's modulus, $v$ is Poisson's ratio, $\lambda=$ $\frac{2 G}{1-2 v}$ is Lame constant, and $\varepsilon_{v}=\partial u_{x} / \partial x+\partial u_{y} / \partial y+\partial u_{z} / \partial z$ is volumetric strain.

The equilibrium equation of rock described with displacement may be obtained by combining Eqs. (1-4)

$G u_{i, j j}+(\lambda+G) \varepsilon_{v, i}+p_{i}=0$.

When groundwater is pumping, not only the fluid flow has seepage velocity, but also solid granules have seepage velocity. So, the seepage velocity of fluid flow can be described as

$V_{\mathrm{f}}=V_{\mathrm{r}}+V_{\mathrm{s}}$,

where $V_{\mathrm{f}}$ is the absolute velocity of fluid flow, and $V_{\mathrm{s}}$ is the absolute velocity of solid granules,

$\nabla V_{\mathrm{s}}=\nabla\left(\frac{\partial u}{\partial t}\right)=\nabla\left(\frac{\partial \nabla u}{\partial t}\right)=\frac{\partial \varepsilon_{v}}{\partial t}$

$V_{\mathrm{r}}$ is relative velocity of fluid flow to solid granules and can be described as

$V_{\mathrm{r}}=-\frac{k}{n \mu} \operatorname{grad}(p)$,

where $k$ is hydraulic conductivity, $n$ is porosity, and $\mu$ is kinetic viscosity.

The hydraulic conductivity of the porous medium can be related to the porosity as (Kozeny 1927; Carman 1937)

$\frac{k}{k_{0}}=\frac{1}{1+\varepsilon_{v}}\left(1+\frac{\varepsilon_{v}}{n_{0}}\right)^{3}$,

where $k_{0}$ is the initial hydraulic conductivity, and $n_{0}$ is initial porosity.

The continue equation of porous media water flow can be described as

$\nabla\left[\rho_{\mathrm{f}} n V_{\mathrm{f}}\right]+\frac{\partial\left[\rho_{\mathrm{f}} n\right]}{\partial t}=0$

The continue equation of solid granules can be described

$\nabla\left[\rho_{\mathrm{s}}(1-n) V_{\mathrm{s}}\right]+\frac{\partial\left[\rho_{\mathrm{s}}(1-n)\right]}{\partial t}=0$

It can be obtained by simplifying (10) and (11)

$\rho_{\mathrm{s}}(1-n) \nabla V_{\mathrm{s}}+(1-n) \frac{\partial \rho_{\mathrm{s}}}{\partial t}-\rho_{\mathrm{s}} \frac{\partial n}{\partial t}=0$

$\rho_{\mathrm{f}} n \nabla V_{\mathrm{r}}+n \rho_{\mathrm{f}} \nabla V_{\mathrm{s}}+n \frac{\partial \rho_{\mathrm{f}}}{\partial t}+\rho_{\mathrm{f}} \frac{\partial n}{\partial t}=0$

We can obtain by dividing $\rho_{\mathrm{f}}$ and $\rho_{\mathrm{s}}$, and then add (12) and (13) $n \nabla V_{\mathrm{r}}+\nabla V_{\mathrm{s}}+\frac{n}{\rho_{\mathrm{f}}} \frac{\partial \rho_{\mathrm{f}}}{\partial t}+\frac{(1-n)}{\rho_{\mathrm{s}}} \frac{\partial \rho_{\mathrm{s}}}{\partial t}=0$

The state equation of fluid can be described in equal temperature as ( $\mathrm{Li}$ et al. 2003)

$\rho_{\mathrm{f}}=\rho_{0} \mathrm{e}^{\left[\beta_{\mathrm{f}}\left(p-p_{0}\right)\right]}$

We can also obtain the state equation of solid granules as

$\rho_{\mathrm{s}}=\rho_{0} \mathrm{e}^{\left[\beta_{\mathrm{s}}\left(p-p_{0}\right)\right]}$

We can obtain equation by integrate $t$ as

$\frac{1}{\rho_{\mathrm{f}}} \frac{\partial \rho_{\mathrm{f}}}{\partial t}=\beta_{\mathrm{f}} \frac{\partial p}{\partial t}$

The same as solid granules

$\frac{1}{\rho_{\mathrm{s}}} \frac{\partial \rho_{\mathrm{s}}}{\partial t}=\beta_{\mathrm{s}} \frac{\partial p}{\partial t}$

where $\beta_{\mathrm{f}}$ is the compressibility coefficient of porosity flow, and $\beta_{\mathrm{s}}$ is the compressibility coefficient of solid granules.

We can obtain equation by combining Eqs. (14-17)

$\nabla\left(-\frac{k}{\mu} \nabla p\right)+\frac{\partial \varepsilon_{v}}{\partial t}+\left(\beta_{\mathrm{s}}(1-n)+n \beta_{\mathrm{f}}\right) \frac{\partial p}{\partial t}=0$

In the case of a porous medium, the change in the porosity due to the change of water pore pressure and soil stress can be expressed by (7), (12) and (18)

$\frac{\partial n}{\partial t}=(1-n)\left(\frac{\partial \varepsilon_{v}}{\partial t}+\beta_{\mathrm{s}} \frac{\partial p}{\partial t}\right)$.

The mathematical model of fluid-solid interaction could be described by four nonlinear partial differential equations (5), (9), (19) and (20) with four dependent variables, $p, u, k$ and $n$.

Finite element approximations

Equations (5), (9), (19) and (20) constitute a general mathematical statement of the physical problem of land subsidence due to pressure decline. Analytical solutions for this general system are not available except for simplified cases. Therefore, numerical approaches may be used to solve general solutions for this problem. The Galerkin finite element method (Thomée 2006) is chosen here owing to its ability to treat compound and complex geometries. In finite element method, a trial solution of the dependent variable $\phi$, which represents $p, u_{x}, u_{y}$ and $u_{z}$ can be expressed by the basic functions as

$\phi \approx \widehat{\phi}=\sum_{L}^{N} \phi_{\mathrm{L}}(t) N_{\mathrm{L}}(x, y, z)$, 
where $N_{\mathrm{L}}(x, y, z)$ is the basis function, $\phi_{\mathrm{L}}(t)$ is the value of the unknown variable, and $N$ is the total number of nodes.

Applying the Galerkin's approach and Green's theorem to Eqs. (5) and (19) yields

$$
\begin{aligned}
& \int_{\mathrm{R}}\left[-\nabla N_{\mathrm{L}} \frac{K}{\mu} \nabla\left(P-G_{\mathrm{s}}\right)+N \frac{\partial \varepsilon_{v}}{\partial t}+N_{\mathrm{L}}\left(\beta_{\mathrm{s}}(1-n)+n \beta_{\mathrm{f}}\right) \frac{\partial P}{\partial t}\right] \mathrm{d} R \\
& =-\int_{\mathrm{B}} N_{\mathrm{L}} \frac{K}{\mu} \nabla P \cdot n \mathrm{~d} B
\end{aligned}
$$

$$
\begin{gathered}
\int_{\mathrm{R}}\left[G\left(\frac{\partial u_{i}}{\partial x_{i}} \frac{\partial N_{\mathrm{L}}}{\partial x_{i}}+\frac{\partial u_{j}}{\partial x_{j}} \frac{\partial N_{\mathrm{L}}}{\partial x_{j}}\right)+(\lambda+G) \frac{\partial N_{\mathrm{L}}}{\partial x_{j}} \varepsilon_{v}+\alpha \frac{\partial N_{\mathrm{L}}}{\partial x_{j}} P\right] \mathrm{d} R \\
=\int_{\mathrm{B}} N_{\mathrm{L}}\left[\left(u_{i}+u_{j}\right)+(\lambda+G) \varepsilon_{v}+\alpha P\right] \cdot n \mathrm{~d} B, \quad i, j=x, y, z
\end{gathered}
$$

where $R$ is the region of interest with boundary $B$, and $n$ is the outward unit vector.

Initial values of pressure head and displacements must be prescribed in the entire region of interest $R$. The boundary conditions associated with equation are of the following types (Huyakorn et al. 1986): Dirichlet type used in pressure head and displacement; Neumann type used in normal flux to Darcy velocity, to solve the nonlinear problem associated with changes in the hydraulic properties due to pore water pressure decreases and solid matrix deformation at each time step, for which the incremental Picard scheme is adopted. For the next iteration level, the hydraulic properties are updated to compute the coefficient matrix and the dependent variables.

\section{Model verification}

A simplified case with known analytical solutions is utilized to verify the finite element model. In this model verification example, a fully penetrating well of radius pumping at a constant rate from a saturated confined aquifer, the aquifer is assumed to be homogeneous and isotropic in space and time. The analytical solutions are derived by Bear and Corapcioglu as follow

$$
\begin{aligned}
& s=-\frac{Q_{\mathrm{w}}}{4 \pi T} W(u) \quad u=\frac{r^{2}}{4 C_{v}}=\frac{S r^{2}}{4 T t} \\
& \Delta z=-\frac{Q_{\mathrm{w}}}{8 \pi C_{v}} W(u) \\
& U_{\mathrm{r}}=-\frac{Q_{\mathrm{w}} t}{4 \pi r B}\left[u W(u)+1-\mathrm{e}^{-u}\right]
\end{aligned}
$$

where $s$ is the drawdown, $\Delta z$ is the vertical displacement at the top surface, $U_{\mathrm{r}}$ is the horizontal displacement, $W(u)=$ $\int_{u}^{\infty} \frac{\mathrm{e}^{-x}}{x} \mathrm{~d} x$ is the well function. $T=K B$ is the transmissivity,
$S$ is the aquifer's storativity, $Q_{\mathrm{w}}$ is pumping rate, and $C_{v}=$ $T / S$ is the consolidation coefficient of the confined aquifer.

As a numerical example, the hydraulic properties of the confined aquifer are given as follows: The initial hydraulic head is assigned to be equal to 0 everywhere in model. A confined aquifer with $K=6.69 \times 10^{-5} \mathrm{~m} / \mathrm{s}, B=142 \mathrm{~m}$, $Q_{\mathrm{w}}=0.5 \mathrm{~m}^{3} / \mathrm{s}, C_{\mathrm{v}}=6 \times 10^{3} \mathrm{~cm}^{2} / \mathrm{s}$.

As shown in Fig. 1, the numerical solutions are demonstrated by circles. Analytical solutions for the drawdown, horizontal and vertical displacements are indicated by solid lines. The relationships of the drawdown, displacement versus the distance from the well after continuous pumping for 3 years are shown in Fig. 1a. The relationship of the drawdown and displacement versus time at the distance of $3 \mathrm{~km}$ from the pumping well is shown in Fig. 1b. Results show that the simulated drawdown and displacements match well with those of the analytical solutions.

\section{Model application}

The proposed model is applied to an ideal three layers numerical experiment. The purpose of the numerical experiments is to analyze the nonlinear effects of hydraulic properties in the seepage and consolidation coupling phenomena. For such a case, the initial pressure head in numerical model is set equal to $0 \mathrm{~m}$. The land surface, i.e., the top boundary, is set free to move both vertically and horizontally. At the bottom boundary, the soil is restrained from both horizontal and vertical movements. The no-flow boundary condition is assigned on the right side and bottom side of the model region. The initial displacement is equal to $0 \mathrm{~m}$ throughout the model. The boundary displacements in the $x$ direction on the two sides located at $x=0$ and $x=1,000$ are constrained. A 90 -m-thick three layers aquifer system with a radial extent of $1,000 \mathrm{~m}$ is considered in this case as shown in Fig. 2. The first aquifer system is a 30 -m-thick phreatic aquifer, and the middle aquifer system is a $10-\mathrm{m}$-thick aquitard aquifer, and the third layer is a 50 -m-thick confined aquifer. The pumping well is drilled vertically through the confined aquifer and the pumping rate is set to $-6 \times t / 360$ at $x=0$. The hydraulic properties parameters of three aquifers are obtained from these references (Kim and Parizek 2005; Biot 1955; Leake and Hsieh 1997; Wang 2000; Hsieh 2006) and used in model as shown in Table 1.

The trend of porosity and hydraulic conductivity around the pumping well at the confined aquifer is shown in Fig. 3. The hydraulic conductivity and porosity dramatically decrease along time and slightly decrease along distance from pumping well. After 10 years of pumping, the hydraulic conductivity with NHP model is estimated to be $0.88 \mathrm{k} 0$, and the porosity with NHP model is about $0.95 \mathrm{n} 0$. 

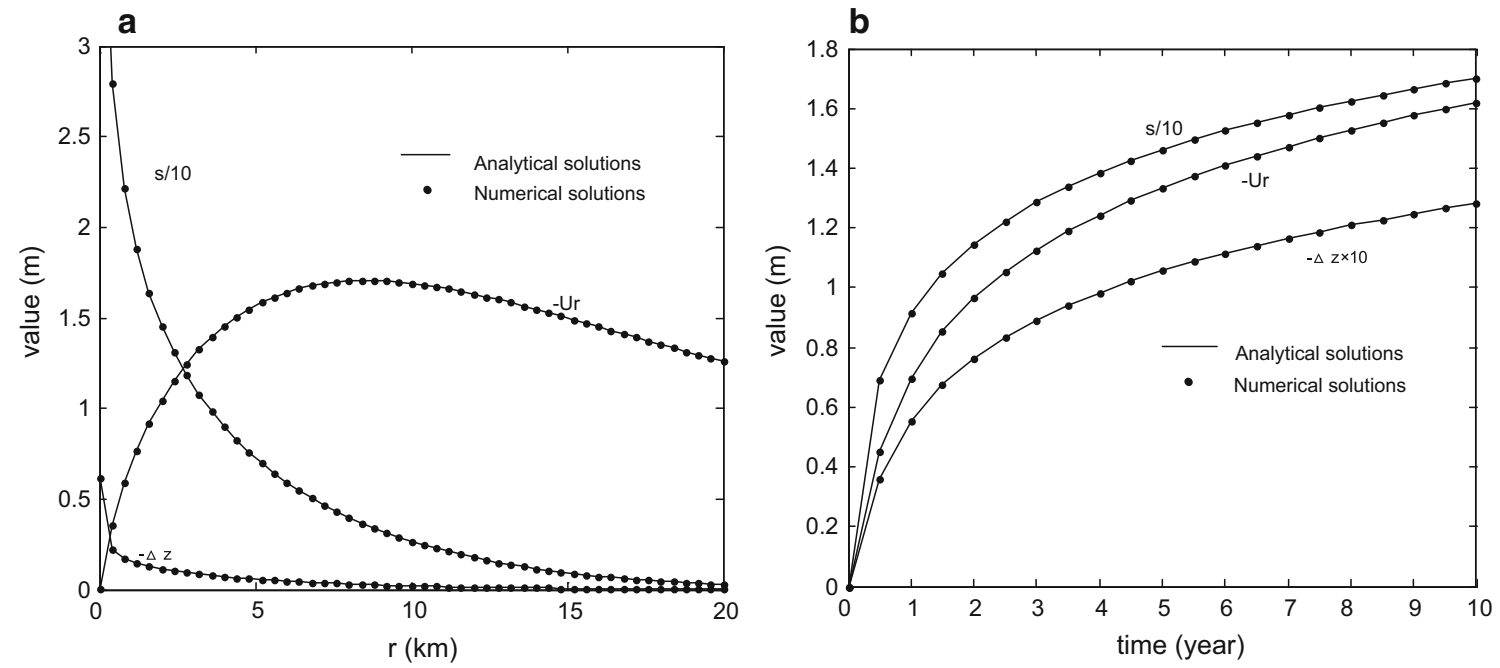

Fig. 1 Spatial distributions (a left) and temporal change (b right) of drawdown, vertical displacement and horizontal displacement in the confined aquifer

Fig. 2 Constant condition of numerical coupling model

Table 1 Basic parameters

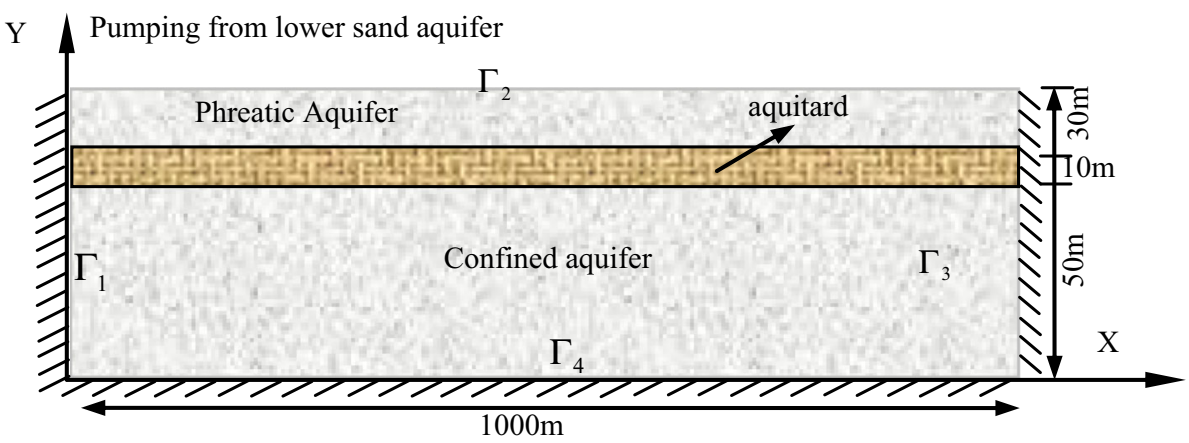

\begin{tabular}{llll}
\hline Parameters & Phreatic aquifer & Aquitard & Confined aquifer \\
\hline Initial porosity $n_{0}($ dimensionless) & 0.4 & 0.5 & 0.3 \\
Viscosity coefficient $\mu(\mathrm{MPa})$ & $2 \times 10^{4}$ & $1.5 \times 10^{4}$ & $1.5 \times 10^{4}$ \\
Acceleration of gravity $g\left(\mathrm{~m} / \mathrm{s}^{2}\right)$ & 9.81 & 9.81 & 9.81 \\
Elastic modulus $E(\mathrm{MPa})$ & 3.6 & 2.6 & 3.6 \\
Shear modulus $G(\mathrm{MPa})$ & 1.44 & 0.96 & 1.44 \\
Poisson's ratio $v$ & 0.25 & 0.35 & 0.25 \\
Lame constant $\lambda(\mathrm{MPa})$ & $1.724 \times 10^{4}$ & $1.624 \times 10^{4}$ & $1.824 \times 10^{4}$ \\
The first biot modulus $H(\mathrm{MPa})$ & $3.42 \times 10^{4}$ & $2.42 \times 10^{4}$ & $3.82 \times 10^{4}$ \\
The second biot modulus $R(\mathrm{MPa})$ & $2.35 \times 10^{4}$ & $1.35 \times 10^{4}$ & $2.95 \times 10^{4}$ \\
Initial hydraulic conductivity $k_{0}[\mathrm{~m} / \mathrm{d}]$ & 25 & 0.01 & 35 \\
Initial pressure $p_{0}(\mathrm{MPa})$ & 1.0 & 1.0 & 1.0 \\
The compressibility of water $\left(\mathrm{GPa}^{-1}\right)$ & 0.5 & 0.5 & 0.4 \\
The compressibility of soil $\left(\mathrm{MPa}^{-1}\right)$ & 0.3 & 0.2 & 0.25
\end{tabular}

The nonlinear hydraulic properties (NHP) are considered in coupling model and the numerical solutions with and without the NHP are shown in Figs. 4, 5 and 6. The result of pressure head with the NHP significantly increases near the pumping well, but slightly decreases from the distance of pumping well compared to the numerical solutions without the NHP model. After 10 years of pumping, the pressure head dropped from -35 to $-50 \mathrm{~m}$ 

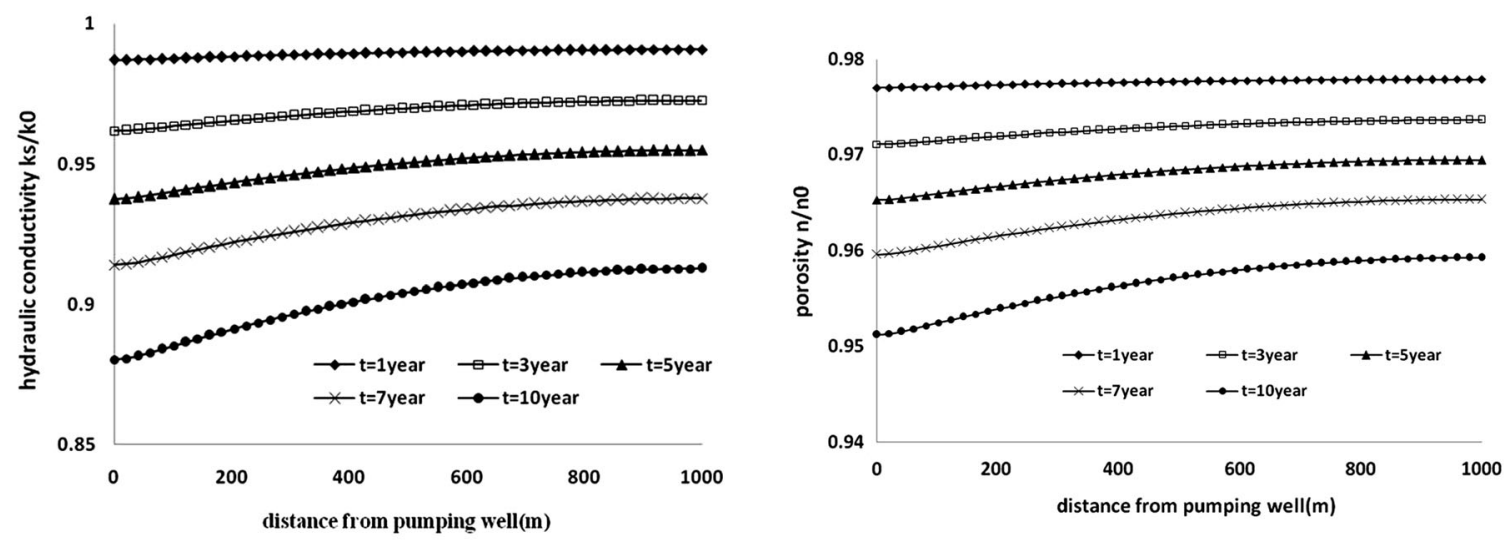

Fig. 3 The special distribution of hydraulic conductivity (left) and porosity (right) at the confined aquifer

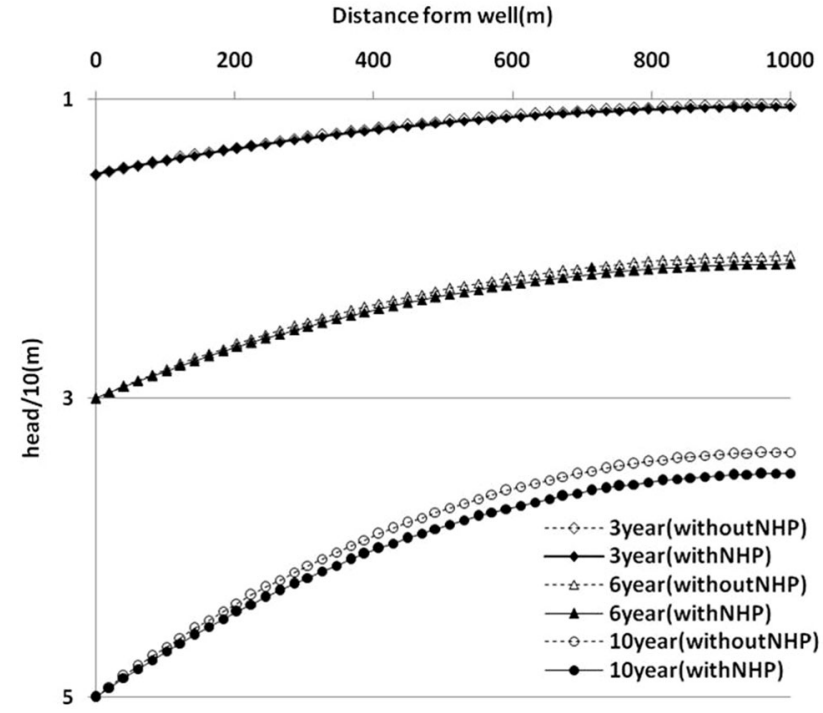

Fig. 4 The distribution of head with and without NHP model at the confined aquifer

with NHP model, but it did from -33.6 to $-50 \mathrm{~m}$ without NHP model. The drawdown is $15 \mathrm{~m}$ with NHP model, whereas it is $16.4 \mathrm{~m}$ without NHP model. Pressure head at $r=500 \mathrm{~m}$ is about $-38.4 \mathrm{~m}$ with the NHP model, whereas about $-37.3 \mathrm{~m}$ without NHP model. The vertical and horizontal displacements are larger with NHP model than without NHP model. The vertical displacement at $r=500 \mathrm{~m}$ is about $-1.57 \mathrm{~cm}$ with NHP model, but about $-1.47 \mathrm{~m}$ without NHP model after 10 years pumping. The horizontal displacement at $r=200 \mathrm{~m}$ is about $-8.14 \mathrm{~m}$ with NHP model, but about $-7.68 \mathrm{~m}$ without NHP model after 10 years pumping. The reason is that the water level decline due to groundwater withdrawal induces the soil compression and the porosity and permeability decrease. Decrease of permeability in regions adjacent to the

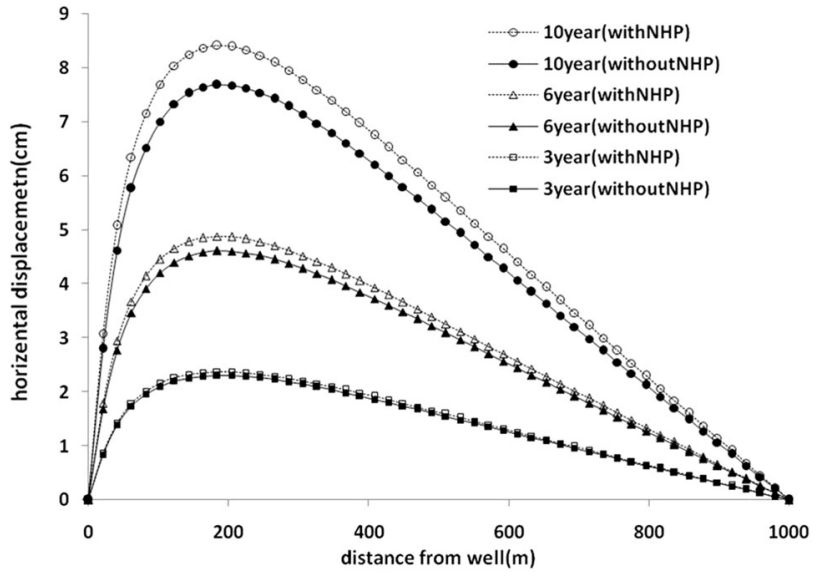

Fig. 5 The distribution of horizontal displacement with and without NHP model at the confined aquifer

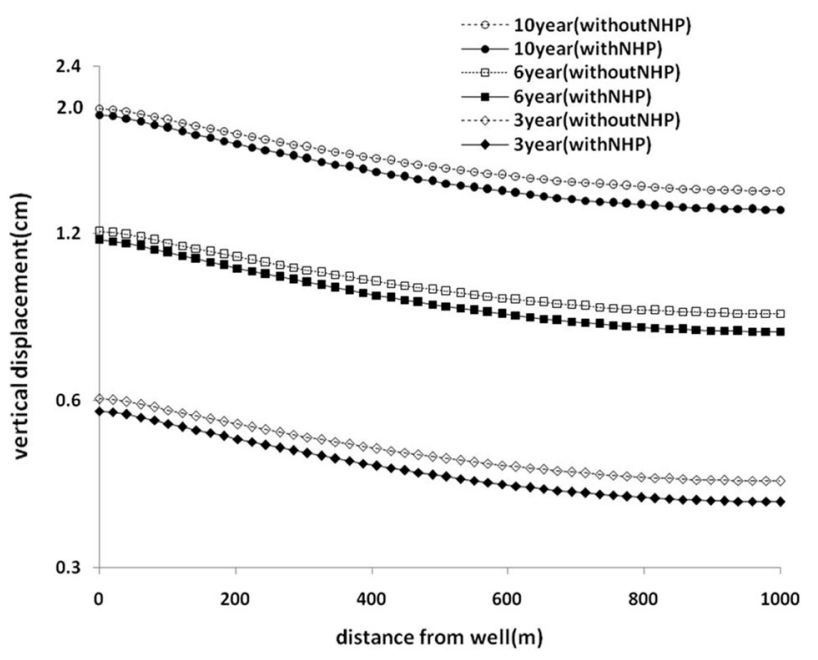

Fig. 6 The distribution of vertical displacement with and without NHP model at the confined aquifer 
Fig. 7 The contour distributions of horizontal displacement without (dashed lines) and with NHP (solid lines) model at the profile
Fig. 8 The contour distributions of vertical displacement without (dashed lines) and with NHP (solid lines) model at the profile
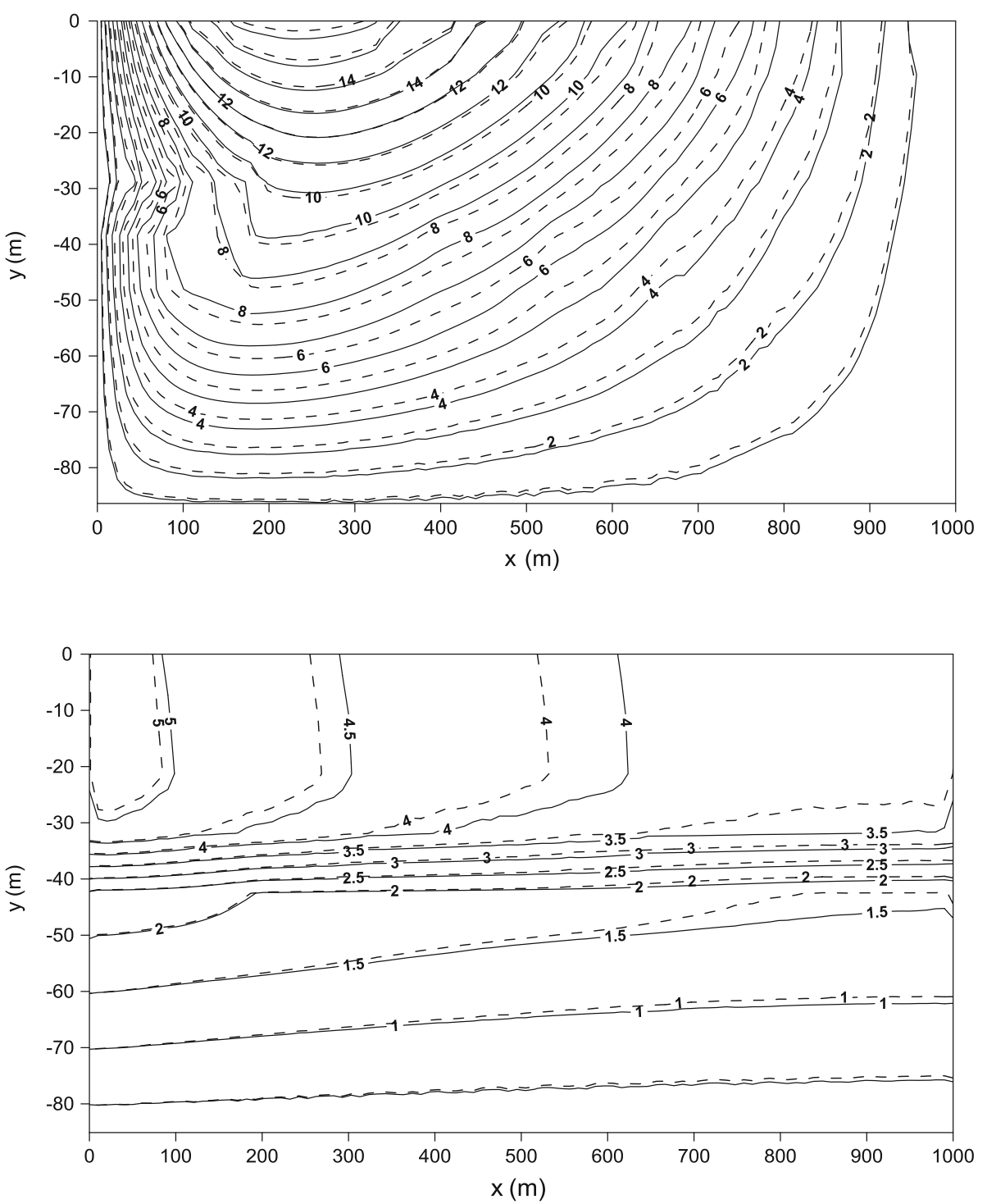

pumping well produces hydraulic gradient and seepage forces that may result in accelerated subsidence. Therefore, the effect of the NHP is an interaction process of pore water pressure and soil consolidation deformation and should not be ignored.

The contour distributions of horizontal and vertical displacements after 10 years of pumping are shown in Figs. 7 and 8. The solid lines stand for the displacement with NHP model, and the dashed lines describe the displacement without NHP model. The max horizontal displacement shows up at about $r=200 \mathrm{~m}$ from the pumping well and after that the horizontal displacement went small and the value is 0 at $r=1,000 \mathrm{~m}$. The reason is that the boundary of the model is restricted and cannot take place lateral deformation. The displacement around pumping well is obvious and the value is smaller along distance from pumping well. The horizontal displacements change dramatically in the aquitard than the other two aquifers. The peak value is reached around $r=250 \mathrm{~m}$ from pumping well in the phreatic aquifer, whereas its value is decreased along vertical depth. The maximum of horizontal displacement is easy to achieve near pumping well. After 10 years of pumping, the horizontal displacement is $12.94 \mathrm{~cm}$ at $(200,-20)$ with NHP model, but the value is $11.93 \mathrm{~cm}$ at $(200,-20)$ without NHP model. The vertical displacement is $4.82 \mathrm{~cm}$ at $(200,-20)$ with NHP model, but the value is $4.7 \mathrm{~cm}$ at $(200,-20)$ without NHP model. As shown in Fig. 8, the value of vertical displacement is from 0 to $2.5 \mathrm{~cm}$ in 50-m-thick confined aquifer, but it is from 2.5 to $4.5 \mathrm{~cm}$ in $10-\mathrm{m}$-thick aquitard. The reason is that the clay soil layer releases water when pumping in the confined aquifer, but it is hard to be supplied because of its weak permeability. 


\section{Conclusions}

This study considered the characteristics of nonlinear changes in the porosity and the hydraulic conductivity that could occur in the field. The deformations of soil were associated with the water pressure change. To show the effect of nonlinear of hydraulic properties, a mathematical model employing the Galerkin finite element method had been proposed to simulate the deformation dependencies of hydraulic properties due to the water pressure decrease in aquifers. The numerical model was verified by comparing with the analytical solutions in the confined aquifer. Results showed that the simulated drawdown and displacements matched well with those of the analytical solutions. The proposed model was applied to an ideal three layers numerical experiment to analyze the NHP in different soils. The result of pressure head with the NHP significantly increased near the pumping well, but slightly decreased from the distance of pumping well compared to the numerical solutions without the NHP model. The value of displacement was smaller without NHP model than with NHP model. The displacement changed dramatically in the aquitard than the other two aquifers. The effect of the NHP had its own importance not only in the groundwater flow filed, but also in the solid skeleton deformation field, and hence it is obvious and cannot be ignored, especially when the long-term pore water pressure change and soil deformation induced by groundwater pumping. The numerical study demonstrated that the NHP character was more desirable than conventional groundwater theory for solving fully coupled soil-water phenomena associated with fluid flow through deforming media. Further studies of various hydrogeology problems are recommended to consider the heterogeneity concerning different stratigraphic conditions in the field.

Acknowledgments This work was supported by Beijing Science and Technology Commission project "Research on water resources sustainable utilization of new air area in Beijing" (Z131100005613001).

\section{References}

Abidin HZ, Gumilar I, Andreas H, Murdohardono D, Fukuda Y (2013) On causes and impacts of land subsidence in Bandung Basin, Indonesia. Environ Earth Sci 68(6):1545-1553

Bai M, Elsworth D (1994) Modeling of subsidence and stress dependent hydraulic conductivity for intact and fractured porous media. Rock Mech Rock Eng 27(4):209-234

Bear J, Corapcioglu MY (1981) Mathematical model for regional land subsidence due to pumping, 2. Integrated aquifer subsidence equations for vertical and horizontal displacements. Water Resour Res 17:947-958

Biot MA (1941) General theory of three-dimensional consolidation. J Appl Phys 12:144-164
Biot MA (1955) Theory of elasticity and consolidation for a porous anisotropic solid. J Appl Phys 26(2):182-185

Budhu M, Adiyaman B (2010) Mechanics of land subsidence due to groundwater pumping. Int $\mathrm{J}$ Numer Anal Methods Geomech 34(14):1459-1478

Budhu M, Adiyaman B (2013) The influence of clay zones on land subsidence from groundwater pumping. GroundWater 51(1):51-57

Carman PC (1937) Fluid flow through granular beds. Trans Inst Chem Eng 15:150-166

Gambolati G, Teatini P, Baú D, Ferronato M (2000) Importance of poroelastic coupling in dynamically active aquifers of the Po river basin, Italy. Water Resour Res 36(9):2443-2460

Gatmiri B, Delage P (1997) A formulation of fully coupled thermalhydraulic-mechanical behaviour of saturated porous medianumerical approach. Int $\mathbf{J}$ Numer Anal Methods Geomech 21(3): 199-225

Hsieh PC (2006) A viscoelastic model for the dynamic response of soils to periodical surface water disturbance. Int J Numer Anal Methods Geomech 30(12):1201-1212

Huang H, Wattenbarger RC, Gai XL, Brown WP, Hehmeyer OJ, Wang JL, Long TA (2013) Using a fully coupled flow and geomechanical simulator to model injection into heavy oil reservoirs. Int J Numer Methods Fluids 71(6):671-686

Huyakorn PS, Springer EP, Guvanasen V, Wadsworth TD (1986) A three-dimensional finite-element model for simulating water flow in variably saturated porous media. Water Resour Res 22(13): 1790-1808

Khan AS, Khan SD, Kakar DM (2013) Land subsidence and declining water resources in Quetta Valley, Pakistan. Environ Earth Sci 70(6):2719-2727

Kim JM (2000) A fully coupled finite element analysis of water-table fluctuation and land deformation in partially saturated soils due to surface loading. Int $\mathrm{J}$ Numer Anal Methods Geomech 49:1101-1119

Kim JM, Parizek RR (1999) A mathematical model for the hydraulic properties of deforming porous media. GroundWater 37(4):546554

Kim JM, Parizek RR (2005) Numerical simulation of the Rhade effect in layered aquifer systems due to groundwater pumping shutoff. Adv Water Resour 28:627-642

Kozeny J (1927) Über kapillare Leitung des Wassers im Boden. Sitzungsber Akad Wiss Wien 136:271-306

Leake SA, Hsieh PA (1997) Simulation of deformation of sediments from decline of groundwater levels in an aquifer underlain by a bedrock step. USGS Open File Report

Lewis RW, Schrefler BA (1978) A fully coupled consolidation model of the subsidence of Venice. Water Resour Res 14(2):223-230

Lewis RW, Schrefler BA (1998) The finite element method in the static and dynamic deformation and consolidation of porous media. Wiley, New York

Li PC, Kong XY, Lu DT (2003) Mathematical modeling of flow in saturated porous media on account of fluid-structure coupling effect. J Hydrodyn (In Chinese) 18(4):419-426

Ouria A, Toufigh MM, Nakhai A (2009) An investigation on the effect of the coupled and uncoupled formulation on transient seepage by the finite element method. Am J Appl Sci 4(12):950-956

Ouria A, Toufigh MM, Nakhai A (2011) Nonlinear analysis of transient seepage by the coupled finite element method. Int $\mathbf{J}$ Mech 5(1):35-39

Safai NM, Pinder GF (1979) Vertical and horizontal land deformation in a desaturating porous medium. Adv Water Resour 2:19-25

Schrefler BA, Zhan XY (1993) A fully coupled model for water flow and airflow in deformable porous media. Water Resour Res 29(1):155-167 
Terzaghi K (1943) Theoretical Soil Mechanics. Wiley, New York

Thomée V (2006) Galerkin finite element methods for parabolic problems, 2nd edn. Springer, Berlin

Wang HF (2000) Theory of linear poroelasticity with application to geomechanics and hydrogeology. Princeton University Press, Princeton

Xue Q, Feng XT, Liu JJ (2005) Study on multi-field coupled model and numerical simulation of landfill gas transport. J Syst Simul (In Chinese) 17:20-24

Yeh HD, Lu RH, Yeh GT (1996) Finite element modeling for land displacements due to pumping. Int J Numer Anal Methods Geomech 20(2):79-99
Zhang CH, Zhao QS, Yu YJ (2011) Model of coupled gas flow and deformation process in heterogeneous coal seams and its application. J Coal Sci Eng 17:76-80

Zimmerman RW (2000) Coupling in poroelasticity and thermoelasticity. Int J Rock Mech Min Sci 37:79-87

Zimmerman RW, Somerton WH, King MS (1986) Compressibility of porous rock. J Geophys Res 91:12765-12777 